\title{
LEARNING STYLES BASED ADAPTIVE INTELLIGENT TUTORING SYSTEMS: DOCUMENT ANALYSIS OF ARTICLES PUBLISHED BETWEEN 2001. AND 2016.
}

\author{
M.tech Amit Kumar, Ph.D. Candidate, School of Computer Science and Engineering, University of Petroleum \\ and Energy Studies, Dehradun, Uttarakhand, India \\ Email: amitanit007@gmail.com \\ M.tech Ninni Singh, Ph.D. Candidate, School of Computer Science and Engineering, University of Petroleum \\ and Energy Studies, Dehradun, Uttarakhand, India \\ Email: ninnisingh1991@gmail.com \\ Dr. Neelu Jyothi Ahuja, School of Computer Science and Engineering, University of Petroleum and Energy \\ Studies, Dehradun, Uttarakhand, India \\ Email: neelu@ddn.upes.ac.in
}

\section{AR T I C LE IN F O}

Original Article

Received: August, 09.2017.

Revised: November, 11.2017.

Accepted: December, 09.2017.

doi:10.5937/IJCRSEE1702083K

UDK

159.953.5.072-057.874

Keywords:

learning styles,

adaptive intelligent tutoring system,

adaptivity,

learner characteristics,

cognitive skills.

\section{A B S T R A C T}

Actualizing instructional intercessions to suit learner contrasts has gotten extensive consideration. Among these individual contrast factors, the observational confirmation in regards to the academic benefit of learning styles has been addressed, yet the examination on the issue proceeds. Late improvements in web-based executions have driven researchers to re-examine the learning styles in adaptive tutoring frameworks. Adaptivity in intelligent tutoring systems is strongly influenced by the learning style of a learner. This study involved extensive document analysis of adaptive tutoring systems based on learning styles. Seventy-eight studies in literature from 2001 to 2016 were collected and classified under select parameters such as main focus, purpose, research types, methods, types and levels of participants, field/area of application, learner modelling, data gathering tools used and research findings. The current studies reveal that majority of the studies defined a framework or architecture of adaptive intelligent tutoring system (AITS) while others focused on impact of AITS on learner satisfaction and academic outcomes. Currents trends, gaps in literature and ications were discussed.

(C) 2017 IJCRSEE. All rights reserved.

\section{INTRODUCTION}

With the growth and advancement in internet technology there has been rapid progress in education delivery employing e-learning mode as (Chrysafiadi. K and Virvou. M, 2012). The motivation of e-learning is not just conveying learning material to the prospective learner on web, but also to cater to the needs of instructors, and students/ learners, who are looking for developing their own subject specific repositories. E-learning offers education to different learners, without

Corresponding Author

M.tech Amit Kumar, University of Petroleum and Energy Studies, Dehradun, Uttarakhand, India Email: amitanit007@gmail.com

\section{c) (i) $\Theta$}

This work is licensed under a Creative Commons Attribution - NonCommercial - NoDerivs 4.0. The article is published with Open Access at www.ijcrsee.com probing into their learning preferences, needs and their knowledge level. Each individual is different and hence the learning process followed is significantly different from the other individual. There are diverse learning traits which influence the learning procedure and to a greater degree determine the learning style of the learner. Several studies have been carried out around these differences with an aim to improve the effectiveness of learning, while designing the teaching and learning environment (Bozkurt and Aydogdu, 2009; Demirbaş and Demirkan, 2003). Numerous instructive hypotheses and studies guarantee that learning gain can be improved, when the learner's learning style is recognized. when compared to the real time class room teaching, there are several shortcomings in the traditional web-based and standalone education systems, such as lack of adaptability, flexibility support and feedback, absence of synergistic support amongst learners and the tutoring system (Xu, 
Wang and $\mathrm{Su}, 2002)$. This is the reason for growing interest of the researchers towards adaptivity in e-learning, ultimately towards the goal of providing benefits to learner (Schiaffino, Garcia, and Amandi, 2008).

Adaptivity is important in learning process to provide and manage learning route adapted for each user, monitoring and interpreting user activities as per their needs and learning preferences. Adaptive feature of elearning framework matches the activities, and personality of the learner with learning material (Park and Lee, 2003). According to Felder and Silverman (1988), many researchers believe that tutoring can be very effective and influential by coordinating the learning material with the learner's preferences and their learning styles. We identified a strong relationship between learning style and adaptive tutoring system. Different learners have different way of learning, and each learner prefers to learn in his/her own individual way that best suits as per individual's characteristics such as learner past knowledge, learning style, cognitive and intellectual ability and the like. Tutoring as per these individual characteristics of learner, make learning effective and promote use of adaptive tutoring system for improving the learning. To improve performance of learning, the teacher should anticipate which learning style is most adapted to the learner.

Learning style is characterized as the strength, quality and inclination in which individuals get and process data. It alludes that each individual has own particular strategy or set of techniques when learning (Felder, R.M and Spurlin, J. 2005). Learning style does not have a single definition, according to Jonassen and Grabowski (2012), learning style is defined as a connected intellectual style, cognitive ability in the field of learning elated one more level from unadulterated handling capacity. As confirmation of this evacuation, learning styles are generally in view of selfrevealed learning inclinations. For measuring them, instruments are utilized that gather some information about their inclinations. Conversely, subjective styles are distinguished by undertaking applicable measures, which test the genuine capacity or expertise.

James and Blank (1993), characterized learning style, as a condition under which, students most beneficially and suitably observe, process, store and recollect what they aspire to learn. After identification of learners learning style, teachers could design the tutoring strategies and provide learning material according to their learning style (Felder and Silverman
(1988). Index of learning style questionnaire defines the dimensions of learning style on a scale ranging between +11 to -11 (Felder and Silverman, 1988). In addition, Felder questionnaire due to its reliability and validity (Litzinger, Lee and Wise, 2005; Van Zwancnberg, Wilkinson and Anderson, 2000) is widely accepted for use in adaptive intelligent tutoring system (Kuljis and Liu, 2005).

An imperative issue in design of adaptive tutoring system is selection of learning characteristics specifically termed as 'attributes' or objects to be considered. Given different learner characteristics, it is important to examine each and in different learning context to be able to mark its importance for future research studies. Many studies reveal that, adaptive tutoring systems based on learning style are more productive, increase learner fulfillment level, minimize learning time and improve academic achievement of learner (Tseng, Chu, Hwang, and Tsai, 2008; Graf, Kinshuk and Liu, 2010). Learning style is a significant feature to focus on individual differences while developing an adaptive system (Graf, Liu and Kinshuk, 2009; Liegle and Janicki, 2006).

\subsection{Literature Review}

Recently, there has been a rapid research in the field of online learning hypermedia particularly focused on adaptation and personalization of web-based learning situations. In the recent past, there have been number of studies focused on the preferences and learning style of learner. Learning style theories and their applications have been used in practice in mid-2000 in the different fields (Ozyurt et. al., 2015). Nowadays, many educational institutes, universities prefer teaching using e-learning courses. There is little focus given to the needs, choice, and characteristics of individual learners and therefore, all learners are treated in same manner. The concentration of the present study is intelligent tutoring systems/ adaptive educational frameworks that suit learning styles. As demonstrated in advance, despite the fact that the significance of learning styles in versatile guideline has been addressed, latest research directed in technological improvements has promising ramifications (Shih et. al. 2008). Work Proposed by Stash, N. (2007) also studied the impact of learning style specifically on adaptive hypermedia systems. The results section of the proposal presents quantified evaluation 
on significant improvement of user/learner's interest and understanding of specific content.

To build our comprehension about existing patterns in adaptive tutoring systems, to put together our contemplates based on learning style and to distinguish conceivable crevices to be returned to in future work, we directed a substance investigation of distributed learning style models and executions on intelligent tutoring system based on learning styles.

We trust that the present investigation adds to the field, prompts more powerful intelligent tutoring system activities, and encourages instructional creators to adapt well to the dynamic learning content creation for academically intelligible e-learning. In the following segment, inquiry and examination methods are compressed trailed by the discoveries of the detailed documents investigation, and conceivable bearings for additionally contemplates. This analysis anticipates to add to the decisions of the present circumstances, and the sorts of studies that are required in this area, its application, the impact of learning style on AITS, and the current slants in the field. Moreover, it has a huge investigation, it plans to decide the present patterns about an adaptive intelligent tutoring system in light of learning styles and existing literature gaps, which will advise for the future investigations. This analysis can add to the improvement of more proficient and gainful AITS. In addition to that, the investigation is relied upon to manage e-learning system developers on the most proficient method to make e-learning with a solid academic foundation. So along with this, the aim of this literature analysis is to do a broad investigation of articles focused on learning styles of learner published from 2001 to 2016 and answer the accompanying research questions.

1 . What are the main focus, purpose, research type, and method of study of AITS addressing learning style?

2 . What are the types of participants and their levels, area/field and data gathering tools employed?

3. What are the learning style applications, and learning style classification algorithms that have been used in AITS?

4. What are the learning styles models accommodated in adaptive intelligent tutoring systems?

5. What are major findings and summary concerning learning styles as an attribute of learner?

\section{MATERIALS AND METHODS}

The current study examines the learning style based AITS, which was conducted through the document analysis published from 2001 to 2016. Document investigation is depicted as systematically organizing, classifying, looking at contents and acquiring outcomes from literature (Ary et. al., 2013). The purpose behind picking the document investigation strategy is that, this joins information which are similar to each other in view of specific ideas, methods, purposes, and applications. A variety of research is being done in this field. Most of them can be classified majorly in three different dimensions such as, Search Strategies, Criteria for Inclusion and Exclusion and finally, Data Accumulation and Investigation. These are being explored in coming subsections.

\subsection{Search Strategy}

There is a drastic growth in the field of adaptive tutoring systems since 1990 (Modritcher, 2008). Extensive document review was conducted over electronic databases including ScienceDirect, GoogleScholar, Dissertations, InderScience, Thesis and SpringerLink. For this keywords namely "personalization", "web-based learning system", "adaptable learning systems", "personalize learning system", "adaptable tutoring system", "adaptation/adaptively learning style", "adaptability", "learning style", "adaptive hypermedia", "adaptive instruction", "adaptive behavior", "adaptive instructional system", "learning system /intelligent tutoring system", "user modeling/learner modeling/student modeling", "artificial intelligence" were used. This study is limited only to these keywords.

\subsection{Criteria for Inclusion and Exclusion}

The accompanying incorporation criteria were utilized for the article determination: articles which are more cited in view of learning style were preferred with an attention on mapping of experimental research for the adequacy of an adaptive intelligent tutoring system. Additionally, the articles which were resolved to be AITS works created in view of learning styles were gathered. Among the rehashed articles, just a single article was incorporated into the study. Through the document 
analysis, above 90 research papers were gathered and it was observed that some of them were redundant with respect to a particular framework of learning styles. So under such conditions, the most extensive article based on a similar framework was incorporated while others were barred. The explanation behind the inclusion/exclusion was that these examinations that depended on a similar framework/ item/foundation, are kept separately for isolated investigations. Consequently, superfluous redundancy was stayed away from analysis. The third aspect for inclusion is that the study must concentrate on learner characteristics i.e. learning style. Since some articles were separated that could be ordered as experimental validation of AITS effectiveness, we chose to incorporate hypothetical recommendations of such frameworks likewise, in which it was proposed that further research should concentrate on exact research findings.

\subsection{Data Accumulation and Investigation}

Document analysis covering several articles has been conducted. The information was sorted based on frequencies and rating of articles. The collected literature is reviewed in the light of research questions outlined earlier in this work. In the second phase, findings/outcomes of the study have been discussed, under categories, purpose, research types, methods, participant's types, participant's level, subject area/field and data gathering tools, based on learning style in adaptive tutoring system, application of Learning style and LS classification algorithms, adaptive intelligent tutoring systems accommodating learning styles, findings and summary of each study on AITS based on learning style were detected. This systematic organization of data was undertaken to ensure ease of investigation and drawing meaningful conclusions.

\section{RESULTS}

Over 78 studies and reviews published from 2001 to 2016, on adaptive intelligent tutoring systems and related aspects, have been critically examined. Several of these publications are published in high impact journals such as 'Expert systems with applications', 'Computers in human behavior', 'Educational technology and society' and many more. Different criteria have been identified to group these studies and results have been tabulated. For a meaningful analysis and ease of representation, a set of criteria have been taken as one group and discussed under one heading. Considering criteria, under a group, the total number of studies are grouped, showing their comparison results in tabular formats. In the present paper, five sets of criteria are presented from section 3.1 to 3.5. Each set holds a list of criteria, which have been used to group the studies and represent the comparison quantitatively as percentage.

\subsection{Main focus, purpose, research type, and methods}

Considering the criteria 'main focus' of research, out of a total of over 78 studies $(n=78)$, a total of 63 studies focused on 'adaptivity based on learning style' as their purpose of research, amounting up to $80.77 \%$, whereas rest 15 studies focused on other learner parameters, as their purpose, making up remaining $19.23 \%$. In addition, defining the purpose of the research, 27 of the studies $(34.61 \%)$ focused, on the proposed ITS model, based on the adaptivity. 13 of the studies $(16.66 \%)$ concerned with impact of learning style on the adaptivity of the intelligent tutoring system as their purpose. 12 of the studies $(15.38 \%)$ were about AITS usability/preferability and the learner satisfaction level with the learning contents. 10 of the studies $(12.82 \%)$ have explained the impact of AITS on the academic achievements or learning outcomes. 6 of the studies $(7.69 \%)$ were used in model for automatic learning style prediction. 8 of the studies $(10.25 \%)$ had the purpose for determining the effectiveness of the AITS and 2 of the studies $(2.56 \%)$ used the 'other parameter', concerning the adaptive tutoring system. In inspecting the motivation behind the reviews, the reviews concerned with assessing the learner execution were considered tentatively and hypothetically. In the majority of the study that is in, 47 of the studies in total $(60.25 \%)$, learners were used as participant and learner evaluation was the motivation of the study. In this review both quantitative and subjective information was assembled from the learner. 12 of the studies, in total $(15.38 \%)$ were empirical studies. At last, 'no assessment' was given in case of 19 of the reviews $(24.35 \%)$. Concerning the methods of the reviews, 33 of the reviews (42.31 $\%$ ) used experimental method and 45 of the studies $(57.69 \%)$ used case studies. 
Table 1. Summary of main focus, purpose, research type, and method for the investigated contemplates

\begin{tabular}{|c|c|c|c|}
\hline & & $\begin{array}{l}\text { Frequency } \\
\text { (f) }\end{array}$ & $\begin{array}{c}\text { Percentage } \\
(\%)\end{array}$ \\
\hline \multirow{2}{*}{$\begin{array}{l}\text { Main Focus } \\
\quad(\mathrm{n}=78)\end{array}$} & $\begin{array}{l}\text { Adaptivity based on } \\
\text { learning style }\end{array}$ & 63 & 80.77 \\
\hline & Other & 15 & 19.23 \\
\hline \multirow{7}{*}{$\begin{array}{l}\text { Purpose } \\
(\mathrm{n}=78)\end{array}$} & $\begin{array}{l}\text { Proposed model of ITS } \\
\text { based on Adaptivity }\end{array}$ & 27 & 34.61 \\
\hline & $\begin{array}{l}\text { Learning Style impact on } \\
\text { Adaptivity }\end{array}$ & 13 & 16.66 \\
\hline & $\begin{array}{l}\text { Determining the usability, } \\
\text { learner level of satisfaction }\end{array}$ & 12 & 15.38 \\
\hline & $\begin{array}{l}\text { Impact of adaptive tutoring } \\
\text { system on academic } \\
\text { achievements/ learning } \\
\text { outcomes }\end{array}$ & 10 & 12.82 \\
\hline & $\begin{array}{l}\text { Model for automatic } \\
\text { learning style prediction }\end{array}$ & 6 & 7.69 \\
\hline & Effectiveness of AITS & 8 & 10.25 \\
\hline & Other & 2 & 2.56 \\
\hline \multirow{3}{*}{$\begin{array}{c}\text { Research } \\
\text { type }(\mathrm{n}=78)\end{array}$} & Theoretical & 19 & 24.35 \\
\hline & Empirical studies & 12 & 15.38 \\
\hline & Learner evaluation & 47 & 60.25 \\
\hline \multirow{2}{*}{$\begin{array}{l}\text { Method } \\
(\mathrm{n}=78)\end{array}$} & Experimental & 33 & 42.31 \\
\hline & Case studies & 45 & 57.69 \\
\hline
\end{tabular}

\subsection{Participants type their levels, subject area/field and data gathering tools}

With reference to the second research question, participant's type, their levels, subject area/field, learner modelling, modelling tools, and data gathering tools used in the studies published between the year 2001 and 2016 were examined. Information outline of these discoveries is shown in Table 2.

As displayed in Table 2, considering the 63 participants in experimental review, majority of participants were learners i.e. $(\mathrm{n}=52$; $82.84 \%)$, while only $(\mathrm{n}=7 ; 11.11 \%)$ were teachers/educators and very small number $(\mathrm{n}=4 ; 6.34 \%)$ were both learners and educators. Considering the criteria 'participants level', out of a total of over 73 studies, 53 of the studies $(72.60 \%)$, were conducted at advanced education level or at engineering level. 10 of the studies $(13.72 \%)$, were conducted at the secondary and elementary education level. Remaining, 10 of the studies (19.69\%) were over mixed group of participants belonging to different levels. The studies, whose level is not specified, are not included in this.

When the reviews were assessed regarding the branch of subject area/field, out of a total of over 87 studies, there were $(n=47 ; 54.02$ $\%)$ offered in computer science/engineering making up the highest share. $(\mathrm{n}=11 ; 12.64 \%)$ were offered in Arithmetic, $(n=7 ; 8.1 \%)$ were offered in chemistry, $(n=6 ; 6.89 \%)$ were offered in Management science, $(n=3 ; 3.45 \%)$ were offered in Administration, $(n=2,2.3 \%)$ were offered under Social science, $(n=2$, $2.3 \%$ ) were offered under law and some others were offered where the learning material was miscellaneous domains or was independent of a particular domain.

Considering the criteria 'tools for $\mathrm{dy}$ namic modelling', out of a total of over 34 studies, 9 of the studies $(26.67 \%)$ were conducted in tracking of learner behavior during tutoring, 7 of the studies $(20.58 \%)$ were used in test results, 6 of the studies (17.65\%) were used for examining the learner feedback, 4 of the studies $(11.76 \%)$ were used in analyzing time spent on learning material, 3 of the studies $(8.82 \%)$ were used to identify the individual profile of learner to provide appropriate learning material and 5 of the studies $(15.70 \%)$ were based on mix types of modelling tools. These aspects listed here, such as tracking learner behavior, test result and feedback, time spent on learning material, identifying individual profile have all been considered here as the 'tools' that have been employed for 'dynamic modelling' within the system.

Data gathering tools used in current studies published from 2001 to 2016 were analyzed. It was seen that 134 data gathering tools were used in the current studies. Some articles report utilization of more than one data gathering instrument on account of more than one reason. As presented in Table 2, considering the criteria 'data gathering tools', out of a total of over 134 studies, it has been observed that a largest share of data gathering tools used was learning style inventory or questionnaire. Learning style questionnaire or inventory tools make up to $(\mathrm{n}=55 ; 41.04 \%)$, learning progress test $(n=26 ; 19.40 \%)$ and online questionnaire $(\mathrm{n}=23 ; 17.16 \%)$. In addition to these, system log report $(\mathrm{n}=14 ; 10.45 \%)$, through interview form $(n=7 ; 5.22 \%)$, Cognitive style inventory $(n=7 ; 5.22)$ and other intelligence inventory $(\mathrm{n}=2 ; 1.5 \%)$ tools were employed in this studies. 
Table 2. Summary of participant's type and their levels, learning modelling/tools, subject area/field, and data gathering tools

\begin{tabular}{|c|c|c|c|c|c|c|c|}
\hline & & $\begin{array}{l}\text { Frequency } \\
\text { (f) }\end{array}$ & $\%$ & & & $\begin{array}{l}\text { Frequency } \\
\text { (f) }\end{array}$ & $\%$ \\
\hline \multirow[t]{4}{*}{1.} & $\begin{array}{l}\text { Participants type } \\
(\mathrm{n}=63)\end{array}$ & & & 5. & $\begin{array}{l}\text { Data gathering } \\
\text { tools }(n=134)\end{array}$ & & \\
\hline & Learner & 52 & 82.54 & & $\begin{array}{l}\text { Learning style } \\
\text { inventory } \\
\text { /questionnaire }\end{array}$ & 55 & 41.04 \\
\hline & Teacher/Educator & 7 & 11.11 & & $\begin{array}{l}\text { Learning progress } \\
\text { test }\end{array}$ & 26 & 19.40 \\
\hline & Mixed & 4 & 6.34 & & $\begin{array}{l}\text { Online } \\
\text { questionnaire }\end{array}$ & 23 & 17.16 \\
\hline \multirow[t]{5}{*}{2.} & $\begin{array}{l}\text { Participants level } \\
(\mathrm{n}=73)\end{array}$ & & & & System log report & 14 & 10.45 \\
\hline & $\begin{array}{l}\text { Higher education/ } \\
\text { Engineering }\end{array}$ & 53 & 72.60 & & $\begin{array}{l}\text { Through interview } \\
\text { form }\end{array}$ & 7 & 5.22 \\
\hline & $\begin{array}{l}\text { Secondary } \\
\text { education }\end{array}$ & 6 & 8.22 & & $\begin{array}{l}\text { Cognitive style } \\
\text { inventory }\end{array}$ & 7 & 5.22 \\
\hline & $\begin{array}{l}\text { Elementary } \\
\text { education }\end{array}$ & 4 & 5.5 & & $\begin{array}{l}\text { Other intelligence } \\
\text { inventory }\end{array}$ & 2 & 1.5 \\
\hline & Mixed & 10 & 19.69 & & & & \\
\hline \multirow[t]{3}{*}{3.} & $\begin{array}{l}\text { Learner } \\
\text { Modeling } \\
(\mathrm{n}=59)\end{array}$ & & & 6. & $\begin{array}{l}\text { Subject area/field } \\
(n=87)\end{array}$ & & \\
\hline & Static & 40 & 67.79 & & $\begin{array}{l}\text { Computer science/ } \\
\text { Engineering }\end{array}$ & 47 & 54.02 \\
\hline & Dynamic & 19 & 32.20 & & Arithmetic & 11 & 12.64 \\
\hline \multirow[t]{7}{*}{4.} & $\begin{array}{l}\text { Tools for } \\
\text { dynamic } \\
\text { modelling }(n=34)\end{array}$ & & & & Chemistry & 7 & 8.1 \\
\hline & $\begin{array}{l}\text { Tracking learner } \\
\text { behavior }\end{array}$ & 9 & 26.47 & & $\begin{array}{l}\text { Management } \\
\text { science }\end{array}$ & 6 & 6.89 \\
\hline & Test result & 7 & 20.58 & & Administration & 3 & 3.45 \\
\hline & Learner feedback & 6 & 17.65 & & Social Science & 2 & 2.3 \\
\hline & Time spent & 4 & 11.76 & & Law & 2 & 2.3 \\
\hline & $\begin{array}{l}\text { Learner selection } \\
\text { based on profile }\end{array}$ & 3 & 8.82 & & $\begin{array}{l}\text { Domain } \\
\text { independent/ } \\
\text { Undermined }\end{array}$ & 9 & 10.34 \\
\hline & Mixed & 5 & 14.70 & & & & \\
\hline
\end{tabular}




\subsection{Application of learning style (LS) for developing adaptive tutoring system and learning style classification techniques}

With reference to the third research question, the use of learning style for developing adaptive tutoring system is shown in

table 3. It is also important to note that more than one application is targeted in one paper/ article.

Table 3. Summary of Application of Learning Style in adaptive tutoring system

\begin{tabular}{|c|c|c|}
\hline $\begin{array}{l}\text { Application of } \\
\text { LS } \\
\text { "What is } \\
\text { adapted?" }\end{array}$ & $\begin{array}{l}\text { Article } \\
\text { (f) }\end{array}$ & References \\
\hline $\begin{array}{l}\text { Learning } \\
\text { material/ learner } \\
\text { characteristics }\end{array}$ & 22 & $\begin{array}{l}\text { Ketamo, H. (2003), Alkhuraiji, S., Cheetham, B.,\& Bamasak, O. (2011), } \\
\text { Vassileva, D. (2006), Baldiris, S. et al. (2008), Sun, S., Joy, M., \& } \\
\text { Griffiths, N. (2007), Reategui, E., Boff, E., \& Campbell, J. A. (2008)., } \\
\text { Cabada, R.Z., Barrón Estrada, M.L., \& Reyes García, C.A. (2011)., } \\
\text { Cabada, R.Z et al. (2009), Germanakos et al. (2008), Conlan, O., Dagger, } \\
\text { D., \& Wade, V. (2002)., Jovanovic et al. (2009), Dwivedi, P., \& } \\
\text { Bharadwaj, K.K. (2013), Sun, S., Joy, M., \& Griffiths, N. (2007)., Kelly, } \\
\text { D., \& Tangney, B. (2005), Klašnja-Mili' cevi 'c et al. (2011), Beal, C. R., } \\
\text { \& Lee, H. (2005)., Özyurt et al. (2013b), Sun et al. (2007), Del Corso, D., } \\
\text { Ovcin,E., \& Morrone, G. (2005)., Tseng,J.C.,Chu,H.-C., Hwang,G.-J.,\& } \\
\text { Tsai,C.-C.(2008)., Yang et al. (2013), and Yasir,E.A.M.,\& } \\
\text { Sami,M.S.(2011). }\end{array}$ \\
\hline $\begin{array}{l}\text { Learning media, } \\
\text { contents and } \\
\text { resource format }\end{array}$ & 4 & $\begin{array}{l}\text { Yasir and Sami(2011), Franzonietal.(2008), Kellyand Tangney(2005), } \\
\text { Baldiris et al.(2008) }\end{array}$ \\
\hline $\begin{array}{l}\text { Learner } \\
\text { knowledge }\end{array}$ & 8 & $\begin{array}{l}\text { Alepis et. al. (2008), Mitrovic, Martin and Mayo (2002), Vassileva and } \\
\text { bontchev (2006), Melis and Andres (2005), Mitrovic, A., Koedinger, K. } \\
\text { R., \& Martin, B. (2003), Popanikolaou et al. (2003), Xu and Wang (2006), } \\
\text { Carmona et al. (2008). }\end{array}$ \\
\hline $\begin{array}{l}\text { Recommended } \\
\text { tutoring materials } \\
\text { /pedagogy }\end{array}$ & 8 & $\begin{array}{l}\text { Limongelli et al. (2011), Essaid El. B., E.H.A., and Adnani, El., M. } \\
\text { (2011), Reategui et al. (2008), Franzoni et al. (2008), Latham et al. } \\
\text { (2014), Latham et al. (2012), , Schiaffino et al. (2008), Wang et al. (2008) }\end{array}$ \\
\hline Intelligent game & 2 & Lin et al. (2013), Feldman, J. et al. (2014). \\
\hline $\begin{array}{l}\text { Learner } \\
\text { evaluation and } \\
\text { practice }\end{array}$ & 3 & Wen, D. et.al. (2007), Baldiris, S. et al. (2008), Cabada, R.Z. et. al. (2011). \\
\hline Other/Mixed & 6 & $\begin{array}{l}\text { Sanders et al. (2010), Shute, V. J., \& Towle, B. (2003). Zakrzewska, D. } \\
\text { (2012), Beal, C. R., \& Lee, H. (2005), Xu, D., \& Wang, H. (2006), } \\
\text { Aleven, V., McLaren, B., Roll, R. \& Koedinger, K. (2006). }\end{array}$ \\
\hline
\end{tabular}

As seen in Table 4, a systematic review of the learning style classification techniques, which have been utilized as part of adaptive tutoring systems, is displayed. While some articles have utilized one algorithm for detection/identification of learning style of learner, most others have used combination of algorithm to provide adaptivity based on learning style.

Rule-based is one of the most popular methods that has been used widely and applied by the 17 articles that help researchers to generate learning style rules to provide system adaptivity. Graf et al. (2009) used Felder index of learning style questionnaire and emphasized that active dimension of learning style model is best among four LS dimensions. The second well known strategy is Bayesian networks methodology which is based on Bayes theorem or Bayes hypothesis. Garcia et al. (2007), Schiaffino et al. (2008) and Garcia et al. (2008) use Bayesian network technique that utilizes Bayes condition to estimate the probability that a learner has preference for a specific learning style when given an activity. Over critical review 7 studies applied this technique, while other 5 studies utilized the Naïve Bayes method, which is also the extended form of Bayesian networks method. 5 studies used artificial neural network techniques, 3 studies used decision tree method, 5 studies used Naïve Bayes techniques, 2 of 
the studies used Markov and Reinforcement model, 1 study used Genetic algorithms, and a remainder of 7 of the studies used, other tech- niques or mixed techniques for learning style classification.

Table 4. Summary of Learning Style Classification techniques

\begin{tabular}{|c|c|c|}
\hline $\begin{array}{l}\text { Classification } \\
\text { technique }\end{array}$ & $\begin{array}{l}\text { Article } \\
\text { (f) }\end{array}$ & References \\
\hline Rule- based & 17 & $\begin{array}{l}\text { Scott et al. (2014), Tseng, J.C., Chu, H.-C., Hwang, G.-J., \& Tsai, } \\
\text { C.C.(2008)., Ketamo, H. (2003), Dorça et al. (2013b), Sun, S., Joy, N } \\
\text { Griffiths, N. (2007), Graf, Kinshuk, \& Liu (2008), Latham et al. (201, } \\
\text { Melis, E., \& Andres, E. (2005)., Graf, KinshuN, Maguire, \& Shtern (, } \\
\text { Ray, R. D., \& Belden, N. (2007)., Xu, D., \& Wang, H. (2006)., , Graf } \\
\text { (2009), Sangineto et al. (2008), Romero et al. (2006), Aleven, V., Mc. } \\
\text { B., Roll, R., \& Koedinger, K. (2006). }\end{array}$ \\
\hline $\begin{array}{l}\text { Bayesian } \\
\text { technique }\end{array}$ & 7 & $\begin{array}{l}\text { Aslan, B. G.et al. (2014)., Botsios, S., Georgiou, D., \& Safouris, N. ( } \\
\text { Garcia et al. (2007), Mc Quiggan, S. et al. (2008)., Conati, C. et al. (2 } \\
\text { Garcia et al. (2006), Carmona, C. et al. (2008)., }\end{array}$ \\
\hline $\begin{array}{l}\text { Artificial Neural } \\
\text { Network }\end{array}$ & 5 & $\begin{array}{l}\text { Cabada et al. (2011), Cabada et al. (2009), Fazlollahtabar and Mahdar } \\
\text { (2009), Kelly, D., \& Tangney, B. (2005), Koutsojannis and } \\
\text { Hatzillygeroudis (2003), Villaverde, J. E. et. al. (2006). }\end{array}$ \\
\hline $\begin{array}{l}\text { Decision Tree } \\
\text { method }\end{array}$ & 3 & $\begin{array}{l}\text { Cha, H.J, et al. (2006), Mc Quiggan et al. (2008), Özpolat, E., \& Aka1 } \\
(2009) \text {. }\end{array}$ \\
\hline Naïve Bayes & 5 & $\begin{array}{l}\text { Feldman et al. (2014), Kelly, D. (2008), McQuiggan et al. (2008)., } \\
\text { Zakrzewska (2012), Kelly, D., \& Tangney, B. (2005). }\end{array}$ \\
\hline $\begin{array}{l}\text { Reinforcement } \\
\text { Learning }\end{array}$ & 1 & Dorça et al. (2013), Balasubramanian, V., Margret Anouncia, S.(2016 \\
\hline Markov model & 1 & Cha, H. J. et al. (2006). \\
\hline $\begin{array}{l}\text { Genetic } \\
\text { Algorithms }\end{array}$ & 1 & Chang, Y. C. et al. (2009). \\
\hline Other & 7 & $\begin{array}{l}\text { Read, T. et. al. (2006), Vassileva, D., \& Bontchev, B. (2006), Hong, F } \\
\text { Kinshuk, D. (2004)., Reategui, E. at al. (2008)., Sanders and Bergas: } \\
\text { (2010), Fazlollahtabar, H., \& Mahdavi, I. (2009), Koutsojannis, C., \& } \\
\text { Hatzilygeroudis, I. (2003). }\end{array}$ \\
\hline
\end{tabular}

\subsection{Adaptive intelligent tutoring system accommodating learning style model}

With reference to the fourth research question, 78 studies were examined. Analysis reveals that some of studies have more than one purpose, for example identifying learning styles, learning style classification, listing learning style predictor elements and data gathering tools. Some of the reviews used combination of more than one learning style model, for example both Felder and Kolb learning style models were utilized as a part of some studies. Therefore, these kinds of learning styles are termed as mixed learning styles. With reference to the first research question, 'learning styles used in adaptive intelligent tutoring system', the research review articles published from 2001 to 2016 were analyzed. Critical analysis reveals that Felder-Silverman learning style model was the most popular learning style model used in adaptive intelligent tutoring system. Felder-Silverman learning style $(\mathrm{n}=30,42.85 \%)$, trailed by Kolb $(\mathrm{n}=4$, $14 \%)$, VARK learning style $(n=2)$, and other are $(n=3)$. Apart from these, limited number of studies include Honey and Mumford $(n=3)$, Myers-Briggs type indicator $(n=2)$ used in the analyzed studies. 
Table 5. Summary of Learning Styles models utilized in AITS summarized in analyzed studies

\begin{tabular}{|c|c|c|}
\hline $\begin{array}{l}\text { Learning style } \\
\text { models }\end{array}$ & $\begin{array}{l}\text { Frequency } \\
\text { (f) }\end{array}$ & References \\
\hline $\begin{array}{l}\text { Felder-Silverman } \\
\text { model }\end{array}$ & 30 & $\begin{array}{l}\text { Adetunji, A. \& Ademola, A. (2014), Alkhuraiji, Cheetham, and } \\
\text { Bamasak (2011), Alfonseca, E. et al. (2006), Baldiris, S. et al. (2008), } \\
\text { Alkhuraiji et al. (2011), Lin, C.F., Yeh, Y., Hung, Y.H., \& Chang, R.I. } \\
\text { (2013)., Essalmi et al. (2010), Feldman et al. (2014), Filippidis, S. K. } \\
\text { \& Tsoukalas, I. A. (2009), Franzoni et al. (2008), Garcia, P., } \\
\text { Schiaffino, S., \& Amandi, A. (2008)., Garcia, P., Amandi, A., } \\
\text { Schiaffino, S., \& Campo, M. (2007)., Graf, S., Kinshuk, K.D., \& Liu, } \\
\text { T.-C. (2008)., Graf, Kinshuk, Maguire, \& Shtern (2010), Graf, S. et al. } \\
\text { (2009), Hong, H., \& Kinshuk, D. (2004)., Limongelli, C., Sciarrone, } \\
\text { F.,Temperini,M., \& Vaste,G. (2011)., Huang, E. Y., Lin, S. W., \& } \\
\text { Huang, T. K. (2012)., Hwang, G.-J., Sung, H.-Y., Hung, C.-M., \& } \\
\text { Huang, I. (2013). Kinshuk, Liu, T. C., \& Graf, S. (2009)., Klašnja- } \\
\text { Milic'evic', A., Vesin, B., Ivanovic', M., \& Budimac, Z. (2011)., } \\
\text { Latham, A., Crockett, K., McLean, D., \& Edmonds, B. (2012)., } \\
\text { Jovanovic, Gaševi c, and Devedži 'c (2009), Kelly and Tangney } \\
\text { (2005), (2011), Lin et al. (2013), Sancho et al. (2005), Mahnane et al. } \\
\text { (2013)., Sanders and Bergasa-Suso (2010). }\end{array}$ \\
\hline Kolb Model & 4 & $\begin{array}{l}\text { Akkoyunlu, B. and Soylu, M. Y. (2008), Moura et al. (2013), Lu, H., } \\
\text { Jia, L., Gong, S. H., \& Clark, B. (2007), Manochehr, N. N. (2006). }\end{array}$ \\
\hline VARK model & 2 & Yasir and Sami (2011), Wnah et al. (2008) \\
\hline $\begin{array}{l}\text { Honey and } \\
\text { Mumford Model }\end{array}$ & 3 & $\begin{array}{l}\text { Anthony, P. et al. (2013), Del Corso et al. (2005), Kurilovas et al. } \\
\text { (2014) }\end{array}$ \\
\hline $\begin{array}{l}\text { Myers-Briggs } \\
\text { Types Indicators } \\
\text { (MBTI) }\end{array}$ & 2 & Essaid E. B. et al. (2011), Kim, J., Lee, A., \& Ryu, H. (2013). \\
\hline Other & 3 & $\begin{array}{l}\text { Yang, T.-C., Hwang, G.-J., \& Yang, S.J.-H. (2013), Read, T., Barros, } \\
\text { B., Bárcena, E., \& Pancorbo, J. (2006), Sun, S., Joy, M., \& Griffiths, N. } \\
(2007) \text {. }\end{array}$ \\
\hline
\end{tabular}

\subsection{Findings and rundown of current reviews on AITS in light of learning styles}

With reference to the last research question, discoveries and outline of the reviews on adaptive intelligent tutoring systems distributed from 2001 to 2016, which address the learning style, were analyzed. Considering the discoveries and outlines of the present reviews, estimation of learning style and adaptability in view of learning style, shows high accomplishment levels with all said being done. Besides, it can be reported that there are high positive opinions concerning learner fulfillments and usability with these situations and their impact on academic accomplishments as well as their learning preferences and needs. Overall finding has been classified under ten main titles as shown in Table 6. Seven of these indicate positive prospective towards adaptive tutoring system while rest indicate negative prospective. The positive findings have a largest share $(\mathrm{n}=95 ; 90.47 \%)$ and negative findings are $(\mathrm{n}=12 ; 9.52 \%)$ which is very lim- ited or less. Data summary of the discoveries/ results is displayed in Table 6.

Table 6. Rundown of findings in the analyzed studies

\begin{tabular}{|c|c|c|c|}
\hline & & $\begin{array}{l}\text { Frequency } \\
\text { (f) }\end{array}$ & $\begin{array}{c}\text { Percentage } \\
(\%)\end{array}$ \\
\hline \multirow{6}{*}{$\begin{array}{l}\text { Positive } \\
\text { perspectives } \\
(\mathrm{n}=95)\end{array}$} & $\begin{array}{l}\text { Learner satisfaction, preferences, } \\
\text { usability, and adaptivity based on } \\
\text { learning style }\end{array}$ & 30 & 28.57 \\
\hline & Correct prediction learning style & 13 & 12.38 \\
\hline & $\begin{array}{l}\text { Relationship between learning } \\
\text { style and other learner } \\
\text { characteristics or navigation }\end{array}$ & 8 & 7.62 \\
\hline & Positive effect on learner learning & 18 & 17.14 \\
\hline & $\begin{array}{l}\text { Positive effect on learning } \\
\text { achievements }\end{array}$ & 15 & 14.29 \\
\hline & $\begin{array}{l}\text { Positive effect of learner } \\
\text { modelling }\end{array}$ & 11 & 10.48 \\
\hline \multirow{3}{*}{$\begin{array}{l}\text { Negative } \\
\text { perspectives } \\
(\mathrm{n}=10)\end{array}$} & $\begin{array}{l}\text { No correct prediction of learning } \\
\text { style }\end{array}$ & 5 & 4.76 \\
\hline & $\begin{array}{l}\text { Not making constructive } \\
\text { outcome on academic and } \\
\text { learners achievements }\end{array}$ & 3 & 2.86 \\
\hline & No effect on learner modeling & 2 & 1.90 \\
\hline
\end{tabular}

\section{DISCUSSION AND OUTCOMES}

This study concentrates on the learning style based AITS, which is an innovative 
field of computerized learning at present days. This study is significant, because it puts forward crucial findings regarding the development of multiple areas, such as, adaptive intelligent tutoring systems, types of courses, types of participants, various applications of the field, classifying algorithms and current research trends in the field through literature gaps which opens the door for future studies. This study incorporates different research perspectives, model designs and findings that may be utilized blindly for the development of the learning style based educational system. However, we welcome all the distinct viewpoint, ideas, and personal perspective based on experience in the educational research.

This study does not contribute only in evaluations, but also helps to propose models, designs and frameworks. In addition, this covers the learners and teacher's views in light of the learning style focused on adaptivity for AITS (Klasnja-Milicevic et. al., 2011). Some students or researchers look for different methods and techniques to improve the performance of the e-learning environment, this in turn makes the system easy and efficient. In this way, AITS is developed to support the current educational system and needs the teacher to review for the correct assessment of learner. Getting their views would increase the effectiveness of the intelligent tutoring system, which will make education productive and will also have a great importance in the future studies of this field.

The finding in Section 3.1 reveals that, considering the empirical and theoretical study, the learning style contributes to a vital role for adaptivity in ITS. AITS that accommodates learning styles has a strong impact on academic achievements and learning outcomes. As shown in section 3.2, the learning style questionnaire/survey is mostly used for the identification of the learning style. This approach is suitable for the traditional teaching where it is difficult to analyses the learner's preference. In this sense, this type of survey suffers from disadvantages like, it depends only on the learner's decisions or it may be biased. While many studies reveal that learning styles can change over time and fixing this makes classification incorrect and inaccurate (Vermount, 1998). This section reveals that the highest participants were learners, and belonged to higher education. Section 3.3 reveals that learning style is mostly utilized to: provide adaptive learning materials to the learner, recommend the best suited pedagogy and to provide correct evaluation of the learner to im- prove the effectiveness of the tutoring system.

The contribution of this research finding is not only to be a guide for the adaptive intelligent tutoring system developer, but also serves to improve the effectiveness of the learning style classification algorithms. The finding in Section 3.4 reveals that the automatic learning style prediction algorithms are Rule-Based, Bayesian, Artificial Neural Networks, Decision Tree, Naïve, Reinforcement Learning and Markov Model in which RuleBased was one of the most popular technique for learning style classification.

The above findings also reveal that a lot of studies of ITS based on learning style were published in high impact national and international journals, in-fact some of them were related to proposing new models. Most of the studies focused on the impact of learning styles on education, productivity of learning system, learning gain, their usability, and higher learner satisfaction. The findings in Section 3.5 reveals that, accommodating learning styles in the intelligent tutoring system makes learning easier, effective and increases learner satisfaction levels towards learning. The positive perspective for accommodating this analyzed study based on the learning style in AITS holds the largest share of about $90 \%$, and holds a very limited negative perspective i.e. about $10 \%$.

\section{CONCLUSION AND FUTURE DIRECTIONS}

As many as 78 studies on adaptive intelligent tutoring systems between the period 2001 to 2016 have been critically examined, classified and compared uncovering developments, trends, research gaps and future prospective. It has been concluded that many of the researchers have used Felder-Silverman learning style models and successfully integrated them in educational/tutoring systems for adaptivity. It has been observed that 'learning style' characteristic of learner has been widely explored to make the systems increasingly adaptive.

Analysis of reviews reveals that learning style highly impact on academic accomplishments, learning performance, and learner satisfaction level. This analysis also reveals the selection and evaluation criteria's of the learning style classification algorithms. RuleBased and Bayesian Network algorithms were increasingly used for automatic prediction of learning style of learner. 
Through the findings and discussion of the studies recommendation and future scope have been put forward. Firstly, there is a chance to investigate a fusion of learning style models in adaptive education systems/ tutoring systems. So it is recommended to explore mixed learning style models for implementation of adaptability in educational system. Though Felder learning style model has been widely used, it would be interesting to note how other contemporary models such as Kolb, Honey \& Mumford model have been used and contributed to adaptivity, based on their specific strengths and weaknesses. Additionally, there is an opportunity to apply algorithms for detection of mixed learning styles for improving the adaptivity in tutoring systems. Finally, as a learner attribute, learning style is sufficiently explored, other learner attributes too deserve to be explored and analyzed.

\section{ACKNOWLEDGEMENTS}

This work is being carried out at University of Petroleum and Energy Studies with the reference number SR/CSI/140/2013. The authors thankfully acknowledge the funding support received from Cognitive Science Research Initiative, Department of Science and Technology for the project. The Authors thank the management of University of Petroleum and Energy Studies, for Supporting the work and granting permission to publish it.

\section{Conflict of interests}

The authors declare no conflict of interest.

\section{REFERENCES}

Adetunji, A., \& Ademola, A. (2014). A Proposed Architectural Model for an Automatic Adaptive E-Learning System Based on Users Learning Style. (IJACSA) International Journal of Advanced Computer Science and Applications, 5(4). DOI: 10.14569/IJACSA.2014.050401

Aleven, V., Mclaren, B., Roll, I., \& Koedinger, K. (2006). Toward meta-cognitive tutoring: A model of help seeking with a Cognitive Tutor. International Journal of Artificial Intelligence in Education, 16(2), 101-128. https://dl.acm.org/ citation.cfm?id=1435346

Alfonseca, E., Carro, R. M., Martín, E., Ortigosa, A., \& Paredes, P. (2006). The impact of learning styles on student grouping for collaborative learning: a case study. User Modeling and UserAdapted Interaction, 16(3), 377-401. https://doi. org/10.1007/s11257-006-9012-7

Alkhuraiji, S., Cheetham, B., \& Bamasak, O. (2011, July). Dynamic adaptive mechanism in learning management system based on learning styles.
In Advanced Learning Technologies (ICALT), 2011 11th IEEE International Conference on (pp. 215-217). IEEE. https://doi.org/10.1109/ ICALT.2011.69

Anthony, P., Joseph, N. E., \& Ligadu, C. (2013). Learning how to program in c using adaptive hypermedia system. International Journal of Information and Education Technology, 3(2), 151. DOI: 10.7763/IJIET.2013.V3.254

Akkoyunlu, B., \& Yilmaz-Soylu, M. (2008). A study of student's perceptions in a blended learning environment based on different learning styles. Educational Technology \& Society, 11(1), 183-193. https://www.learntechlib.org/p/75024/

Alepis, E., Virvou, M., \& Kabassi, K. (2008, November). Mobile education: Towards affective bimodal interaction for adaptivity. In Digital Information Management, 2008. ICDIM 2008. Third International Conference on (pp. 51-56). IEEE. DOI: 10.1109/ICDIM.2008.4746737

Ary, D., Jacobs, L. C., Irvine, C. K. S., \& Walker, D. (2013). Introduction to research in education. Cengage Learning. https://www.goodreads. $\mathrm{com} / \mathrm{book} / \mathrm{show} / 3528730$-introduction-to-research-in-education

Aslan, B. G., Öztürk, Ö., \& Inceoglu, M. M. (2014). Effect of Bayesian Student Modeling on Academic Achievement in Foreign Language Teaching (University Level English Preparatory School Example). Educational Sciences: Theory and Practice, 14(3), 1160-1168. https://eric. ed.gov/?id=EJ1034095

Baldiris, S., Santos, O. C., Barrera, C., Boticario, J., Velez, J., \& Fabregat, R. (2008). Integration of educational specifications and standards to support adaptive learning scenarios in ADAPTAPlan. IJCSA, 5(1), 88-107. http://www.tmrfindia.org/ijcsa/V5I16.pdf

Balasubramanian, V., \& Anouncia, S. M. (2016). Learning style detection based on cognitive skills to support adaptive learning environment-A reinforcement approach. Ain Shams Engineering Journal. https://doi.org/10.1016/j. asej.2016.04.012

Beal, C., \& Lee, H. (2005, July). Creating a pedagogical model that uses student self-reports of motivation and mood to adapt ITS instruction. In Workshop on Motivation and Affect in Educational Software, in conjunction with the 12th International Conference on Artificial Intelligence in Education (Vol. 574). https://www. researchgate.net/publication/26621956_Integration of Educational Specifications and Standards to _oupport Ad̄aptive_Learning_Scenarios in ${ }^{-} \bar{D}$ APTAPTan

Bozkurt, O., \& Aydoğdu, M. (2009). A comparative analysis of the effect of dunn and dunn learning styles model and traditional teaching method on 6th grade students' achievement levels and attitudes in science education lesson. Elementary Education Online, 8(3), 741-754. https://www. academia.edu/4805395/A_Comparative_Analysis of the Effect of Dunn and Dunn Learning Styles Model and Traditional Teaching Method on 6th Grade Students A Achievement_Levels and_Attitudes_in_Science_Education Lesson

Botsios, S., Georgiou, D., \& Safouris, N. (2008). Contributions to adaptive educational hypermedia systems via on-line learning style estimation. 
(IJCRSEE) International Journal of Cognitive Research in Science, Engineering and Education

Vol. 5, No. 2, 2017.

Journal of Educational Technology \& Society, 11(2). http://www.ifets.info/journals/11_2/23. pdf

Cabada, R. Z., Estrada, M. L. B., \& García, C. A. R. (2011). EDUCA: A web 2.0 authoring tool for developing adaptive and intelligent tutoring systems using a Kohonen network. Expert Systems with Applications, 38(8), 9522-9529. https://doi. org/10.1016/j.eswa.2011.01.145

Cabada, R., Estrada, M., Sanchez, L., Sandoval, G., Velazquez, J., \& Barrientos, J. (2009). Modeling student's learning styles in web 2.0 learning systems. World Journal on Educational Technology, 1(2), 75-88. https://doi.org/10.1007/978-3642-05258-3 45

Carmona, C., Castillo, G., \& Millán, E. (2008, July). Designing a dynamic bayesian network for modeling students' learning styles. In Advanced Learning Technologies, 2008. ICALT'08. Eighth IEEE International Conference on (pp. 346350). IEEE. http://doi.ieeecomputersociety. org/10.1109/ICALT.2008.116

Conati, C., Gertner, A., \& Vanlehn, K. (2002). Using Bayesian networks to manage uncertainty in student modeling. User modeling and useradapted interaction, 12(4), 371-417. https://doi. org/10.1023/A:1021258506583

Cha, H. J., Kim, Y. S., Park, S. H., Yoon, T. B., Jung, Y. M., \& Lee, J. H. (2006, June). Learning styles diagnosis based on user interface behaviors for the customization of learning interfaces in an intelligent tutoring system. In International Conference on Intelligent Tutoring Systems (pp. 513-524). Springer, Berlin, Heidelberg. https:// doi.org/10.1007/11774303 51

Chang, Y. C., Kao, W. Y., Chu, C. P., \& Chiu, C. H. (2009). A learning style classification mechanism for e-learning. Computers \& Education, 53(2), 273-285. https://doi.org/10.1016/j. compedu.2009.02.008

Chrysafiadi, K., \& Virvou, M. (2012). Evaluating the integration of fuzzy logic into the student model of a web-based learning environment. Expert Systems with Applications, 39(18), 13127-13134. https://doi.org/10.1016/j.eswa.2012.05.089

Dagger, D., Wade, V., \& Conlan, O. (2002). Towards a standards-based approach to e-learning personalization using reusable learning objects. In E-Learn: World Conference on E-Learning in Corporate, Government, Healthcare, and Higher Education (pp. 210-217). Association for the Advancement of Computing in Education (AACE). https://www.scss.tcd.ie/Owen.Conlan/ publications/eLearn2002_v1.24_Conlan.pdf

Demirbas, O. O., \& Demirkan, H. (2003). Focus on architectural design process through learning styles. Design Studies, 24(5), 437-456. https:// doi.org/10.1016/S0142-694X(03)00013-9

Del Corso, D., Ovcin, E., \& Morrone, G. (2005). A teacher friendly environment to foster learnercentered customization in the development of interactive educational packages. IEEE Transactions on Education, 48(4), 574-579. DOI: 10.1109/TE.2005.850709

De Moura, F. F., Franco, L. M., De Melo, S. L., \& Fernandes, M. A. (2013, October). Development of learning styles and multiple intelligences through particle swarm optimization. In $S y s$ tems, Man, and Cybernetics (SMC), 2013 IEEE International Conference on (pp. 835-840).
IEEE. DOI:10.1109/SMC.2013.148

Dorça, F. A., Lima, L. V., Fernandes, M. A., \& Lopes, C. R. (2013). Automatic student modeling in adaptive educational systems through probabilistic learning style combinations: a qualitative comparison between two innovative stochastic approaches. Journal of the Brazilian Computer Society, 19(1), 43-58. DOI 10.1007/s13173012-0078-2

Dwivedi, P., \& Bharadwaj, K. K. (2013). Effective trustaware e-learning recommender system based on learning styles and knowledge levels. Journal of Educational Technology \& Society, 16(4), 201. $\mathrm{http}: / / w w w . i f e t s . i n f o / j o u r n a l s / 16$ 4/16.pdf

Essaid El Bachari, E. H. A., \& El Adnani, M. (2011). E-LEARNING PERSONALIZATION BASED ON DYNAMIC LEARNERS'PREFERENCE. International Journal of Computer Science \& Information Technology (IJCSIT), 3(3), 200-216. https://www.scribd.com/ document/58614290/E-Learning-personalization-based-on-Dynamic-learners-preference

Essalmi, F., Ayed, L. J. B., Jemni, M., \& Graf, S. (2010). A fully personalization strategy of E-learning scenarios. Computers in Human Behavior, 26(4), 581-591. DOI: 10.1016/j.chb.2009.12.010

Fazlollahtabar, H., \& Mahdavi, I. (2009). User/tutor optimal learning path in e-learning using comprehensive neuro-fuzzy approach. Educational Research Review, 4(2), 142-155. https://doi. org/10.1016/j.edurev.2009.02.001

Felder, R. M., \& Silverman, L. K. (1988). Learning and teaching styles in engineering education. Engineering education, 78(7), 674-681. http:// www4.ncsu.edu/unity/lockers/users/f/felder/ public/Papers/LS-1988.pdf

Felder, R. M., \& Spurlin, J. (2005). Applications, reliability and validity of the index of learning styles. International journal of engineering education, 21(1), 103-112. https://www.ijee.ie/ articles/Vol21-1/IJEE1553.pdf

Feldman, J., Monteserin, A., \& Amandi, A. (2014). Detecting students' perception style by using games. Computers \& Education, 71, 14-22. https://doi.org/10.1016/j.compedu.2013.09.007

Filippidis, S. K., \& Tsoukalas, I. A. (2009). On the use of adaptive instructional images based on the sequential-global dimension of the Felder-Silverman learning style theory. Interactive Learning Environments, 17(2), 135-150. http://dx.doi. org/10.1080/10494820701869524

Franzoni, A. L., Assar, S., Defude, B., \& Rojas, J. (2008, July). Student learning styles adaptation method based on teaching strategies and electronic media. In Advanced Learning Technologies, 2008. ICALT'08. Eighth IEEE International Conference on (pp. 778-782). IEEE. DOI: 10.1109/ ICALT.2008.149

García, P., Amandi, A., Schiaffino, S., \& Campo, M. (2007). Evaluating Bayesian networks' precision for detecting students' learning styles. Computers \& Education, 49(3), 794-808. https://doi. org/10.1016/j.compedu.2005.11.017

García, P., Schiaffino, S., \& Amandi, A. (2008). An enhanced Bayesian model to detect students' learning styles in Web-based courses. Journal of Computer Assisted Learning, 24(4), 305-315. DOI: $10.1111 / \mathrm{j} .1365-2729.2007 .00262 . \mathrm{x}$

Germanakos, P., Tsianos, N., Lekkas, Z., Mourlas, C., \& Samaras, G. (2008). Capturing essential intrinsic 
user behaviour values for the design of comprehensive web-based personalized environments. Computers in Human Behavior, 24(4), 14341451. DOI: $10.1016 /$ j.chb.2007.07.010

Graf, S., \& Liu, T. C. (2008, July). Identifying learning styles in learning management systems by using indications from students' behaviour. In $\mathrm{Ad}$ vanced Learning Technologies, 2008. ICALT'08. Eighth IEEE International Conference on (pp. 482-486). IEEE. DOI: 10.1109/ICALT.2008.84

Graf, S., Liu, T. C., Chen, N. S., \& Yang, S. J. (2009). Learning styles and cognitive traits-Their relationship and its benefits in web-based educational systems. Computers in Human Behavior, 25(6), 1280-1289. https://doi.org/10.1016/j. chb.2009.06.005

Graf, S., \& Liu, T. C. (2010). Analysis of learners' navigational behaviour and their learning styles in an online course. Journal of Computer Assisted Learning, 26(2), 116-131. doi: 10.1111/j.13652729.2009.00336.x

Hong, H. (2004). Adaptation to student learning styles in web based educational systems. In EdMedia: World Conference on Educational Media and Technology (pp. 491-496). Association for the Advancement of Computing in Education (AACE). https://www.learntechlib.org/p/12978/

Huang, E. Y., Lin, S. W., \& Huang, T. K. (2012). What type of learning style leads to online participation in the mixed-mode e-learning environment? A study of software usage instruction. Computers \& Education, 58(1), 338-349. https://doi. org/10.1016/j.compedu.2011.08.003

Hwang, G. J., \& Tsai, C. C. (2011). Research trends in mobile and ubiquitous learning: A review of publications in selected journals from 2001 to 2010 . British Journal of Educational Technology, 42(4). DOI: 10.1111/j.1467-8535.2011.01183.x

Hwang, G. J., Sung, H. Y., Hung, C. M., \& Huang, I. (2013). A Learning Style Perspective to Investigate the Necessity of Developing Adaptive Learning Systems. Educational Technology \& Society, 16(2), 188-197. https://eric. ed.gov/?id=EJ1016557

James, W. B., \& Blank, W. E. (1993). Review and critique of available learning-style instruments for adults. New Directions for Adult and Continuing Education, 1993(59), 47-57. https://eric. ed.gov/?id=EJ472130

Jonassen, D. H., \& Grabowski, B. L. (2012). Handbook of individual differences, learning, and instruction. Routledge. https://doi.org/10.1016/00224405(95)00013-C

Jovanović, J., Gašević, D., \& Devedžić, V. (2009). TANGRAM for personalized learning using the semantic web technologies. Journal of emerging technologies in web intelligence, 1(1), 6-21. DOI: $10.4304 /$ jetwi.1.1.6-21

Kelly, D., \& Tangney, B. (2005, July). 'First Aid for You': getting to know your learning style using machine learning. In Advanced Learning Technologies, 2005. ICALT 2005. Fifth IEEE International Conference on (pp. 1-3). IEEE. DOI: 10.1109/ICALT.2005.1

Kelly, D. (2008). Adaptive versus learner control in a multiple intelligence learning environment. Journal of Educational Multimedia and Hypermedia, 17(3), 307. https://www.learntechlib. $\operatorname{org} / \mathrm{p} / 24252$

Ketamo, H. (2003). An adaptive geometry game for handheld devices. Educational Technology \& Society, 6(1), 83-95. http://www.ifets.info/journals/6 1/ketamo.html

Kim, J., Lee, A., \& Ryu, H. (2013). Personality and its effects on learning performance: Design guidelines for an adaptive e-learning system based on a user model. International Journal of Industrial Ergonomics, 43(5), 450-461. https://doi. org/10.1016/j.ergon.2013.03.001

Klašnja-Milićević, A., Vesin, B., Ivanović, M., \& Budimac, Z. (2011). E-Learning personalization based on hybrid recommendation strategy and learning style identification. Computers \& Education, 56(3), 885-899. https://doi.org/10.1016/j. compedu.2010.11.001

Koutsojannis, C., Prentzas, J., \& Hatzilygeroudis, I. (2001). A web-based intelligent tutoring system teaching nursing students fundamental aspects of biomedical technology. In Engineering in Medicine and Biology Society, 2001. Proceedings of the 23rd Annual International Conference of the IEEE (Vol. 4, pp. 4024-4027). IEEE. DOI: 10.1109/IEMBS.2001.1019728

Kurilovas, E., Kubilinskiene, S., \& Dagiene, V. (2014). Web 3.0-Based personalisation of learning objects in virtual learning environments. Computers in Human Behavior, 30, 654-662. https://doi. org/10.1016/j.chb.2013.07.039

Kuljis, J., \& Liu, F. (2005). A Comparison of Learning Style Theories on the Suitability for e-learning. Web Technologies, Applications, and Services, 2005, 191-197. http://www.actapress.com/PaperInfo. aspx?PaperID $=21202 \&$ reason $=500$

Latham, A., Crockett, K., McLean, D., \& Edmonds, B. (2012). A conversational intelligent tutoring system to automatically predict learning styles. Computers \& Education, 59(1), 95-109. https:// doi.org/10.1016/j.compedu.2011.11.001

Latham, A., Crockett, K., \& McLean, D. (2014). An adaptation algorithm for an intelligent natural language tutoring system. Computers \& Education, 71, 97-110. https://doi.org/10.1016/j. compedu.2013.09.014

Liegle, J. O., \& Janicki, T. N. (2006). The effect of learning styles on the navigation needs of Webbased learners. Computers in human behavior, 22(5), 885-898. https://www.researchgate.net/ profile/Jens_Liegle/publication/201381995_Effect of Learning_Styles_on the Navigational Needs_of Computer-Based Training Module ${ }^{-}$

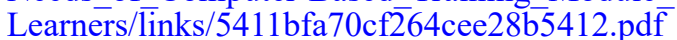

Limongelli, C., Sciarrone, F., Temperini, M., \& Vaste, G. (2011). The lecomps 5 framework for personalized web-based learning: a teacher's satisfaction perspective. Computers in Human Behavior, 27(4), 1310-1320. https://doi.org/10.1016/j. chb.2010.07.026

Lin, C. F., Yeh, Y. C., Hung, Y. H., \& Chang, R. I. (2013). Data mining for providing a personalized learning path in creativity: An application of decision trees. Computers \& Education, 68, 199-210. https://doi.org/10.1016/j.compedu.2013.05.009

Litzinger, T. A., Wise, J. C., \& Lee, S. H. (2005). Selfdirected Learning Readiness Among Engineering Undergraduate Students. Journal of Engineering Education, 94(2), 215-221. https://doi. org/10.1002/j.2168-9830.2005.tb00842.x

Lu, H., Jia, L., Gong, S. H., \& Clark, B. (2007). The relationship of Kolb learning styles, online learning behaviors and learning outcomes. Journal of 
(IJCRSEE) International Journal of Cognitive Research in Science, Engineering and Education

Vol. 5, No. 2, 2017.

Educational Technology \& Society, 10(4). http:// www.ifets.info/journals/10_4/17.pdf

Mahnane, L., Laskri, M. T., \& Trigano, P. (2013). A model of adaptive e-learning hypermedia system based on thinking and learning styles. International Journal of Multimedia and Ubiquitous Engineering, 8(3), 339-350. https://doi. org/10.4018/ijicte.2013100102

Manochehr, N. N. (2006). The influence of learning styles on learners in e-learning environments: An empirical study. Computers in Higher Education Economics Review, 18(1), 10-14. http:// www.economicsnetwork.ac.uk/cheer/ch18/ manochehr.pdf

Mcquiggan, S. W., Mott, B. W., \& Lester, J. C. (2008). Modeling self-efficacy in intelligent tutoring systems: An inductive approach. User modeling and user-adapted interaction, 18(1), 81-123. https://doi.org/10.1007/s11257-007-9040-y

Melis, E., \& Siekmann, J. (2004, June). Activemath: An intelligent tutoring system for mathematics. In ICAISC (pp. 91-101). https://doi.org/10.1007/ b98109

Mitrovic, A., Martin, B., \& Mayo, M. (2002). Using evaluation to shape ITS design: Results and experiences with SQL-Tutor. User Modeling and User-Adapted Interaction, 12(2), 243-279. https://doi.org/10.1023/A:1015022619307

Mitrovic, A., Koedinger, K., \& Martin, B. (2003). A comparative analysis of cognitive tutoring and constraint-based modeling. User Modeling 2003, 147-147. https://doi.org/10.1007/3-54044963-9 42

Mödritscher, F. (2008). Adaptive e-learning environments: theory, practice, and experience. VDM, Müller.

Mustafa, Y. E. A., \& Sharif, S. M. (2011). An approach to adaptive e-learning hypermedia system based on learning styles (AEHS-LS): Implementation and evaluation. International Journal of Library and Information Science, 3(1), 15-28. http:// www.academicjournals.org/journal/IJLIS/article-abstract/75161B52666

Özyurt, Ö., \& Özyurt, H. (2015). Learning style based individualized adaptive e-learning environments: Content analysis of the articles published from 2005 to 2014. Computers in Human Behavior, 52, 349-358. https://doi.org/10.1016/j. chb.2015.06.020

Özpolat, E., \& Akar, G. B. (2009). Automatic detection of learning styles for an e-learning system. Computers \& Education, 53(2), 355-367. https://doi. org/10.1016/j.compedu.2009.02.018

Özyurt, Ö., Özyurt, H., Baki, A., \& Güven, B. (2013). Integration into mathematics classrooms of an adaptive and intelligent individualized e-learning environment: Implementation and evaluation of UZWEBMAT. Computers in Human Behavior, 29(3), 726-738. https://doi.org/10.1016/j. chb.2012.11.013

Papanikolaou, K. A., Grigoriadou, M., Kornilakis, H., \& Magoulas, G. D. (2003). Personalizing the Interaction in a Web-based Educational Hypermedia System: the case of INSPIRE. User modeling and user-adapted interaction, 13(3), 213267. https://doi.org/10.1023/A:1024746731130

Park, O. C., \& Lee, J. (2003). Adaptive instructional systems. Educational Technology Research and Development, 25, 651-684. http://www.aect.org/ edtech/ed1/22/index.html
Ray, R. D., \& Belden, N. (2007). Teaching college level content and reading comprehension skills simultaneously via an artificially intelligent adaptive computerized instructional system. The Psychological Record, 57(2), 201. https://doi. org/10.1007/BF03395572

Read, T., Barros, B., Bárcena, E., \& Pancorbo, J. (2006). Coalescing individual and collaborative learning to model user linguistic competences. User Modeling and User-Adapted Interaction, 16(3), 349-376. https://doi.org/10.1007/s11257006-9014-5

Reategui, E., Boff, E., \& Campbell, J. A. (2008). Personalization in an interactive learning environment through a virtual character. Computers \& Education, 51(2), 530-544. https://doi. org/10.1016/j.compedu.2007.05.018

Romero, C., Ventura, S., Gibaja, E. L., Hervás, C., \& Romero, F. (2006). Web-based adaptive training simulator system for cardiac life support. Artificial Intelligence in Medicine, 38(1), 67-78. https://doi.org/10.1016/j.artmed.2006.01.002

Sancho, P., Martínez, I., \& Fernández-Manjón, B. (2005). Semantic Web Technologies Applied to e-learning Personalization in $<$ e-aula $>$. Journal of Universal Computer Science, 11(9), 14701481. http://dx.doi.org/10.3217/jucs-011-091470

Sanders, D. A., \& Bergasa-Suso, J. (2010). Inferring learning style from the way students interact with a computer user interface and the WWW. IEEE Transactions on Education, 53(4), 613620. https://doi.org/10.1109/TE.2009.2038611

Sangineto, E., Capuano, N., Gaeta, M., \& Micarelli, A. (2008). Adaptive course generation through learning styles representation. Universal Access in the Information Society, 7(1-2), 1-23. https:// doi.org/10.1007/s10209-007-0101-0

Schiaffino, S., Garcia, P., \& Amandi, A. (2008). eTeacher: Providing personalized assistance to e-learning students. Computers \& Education, 51(4), 1744-1754. https://doi.org/10.1016/j.compedu.2008.05.008

Scott, E., Rodríguez, G., Soria, Á., \& Campo, M. (2014). Are learning styles useful indicators to discover how students use Scrum for the first time? Computers in Human Behavior, 36, 5664. https://doi.org/10.1016/j.chb.2014.03.027

Shute, V., \& Towle, B. (2003). Adaptive e-learning. Educational psychologist, 38(2), 105-114. http:// dx.doi.org/10.1207/S15326985EP3802 5

Shih, M., Feng, J., \& Tsai, C. C. (2008). Research and trends in the field of e-learning from 2001 to 2005: A content analysis of cognitive studies in selected journals. Computers \& Education, 51(2), 955-967. https://doi.org/10.1016/j. compedu.2007.10.004

Stash, N. (2007). Incorporating cognitive/learning styles in a general-purpose adaptive hypermedia system. Dissertation Abstracts International, 68(04). https://doi.org/10.1145/1324960.1324963

Sun, S., Joy, M., \& Griffiths, N. (2007). The use of learning objects and learning styles in a multiagent education system. Journal of Interactive Learning Research, 18(3), 381. http://www.dcs. warwick.ac.uk/ nathan/resources/Publications/ edmedia-2005.pdf

Tseng, J. C., Chu, H. C., Hwang, G. J., \& Tsai, C. C. (2008). Development of an adaptive learning system with two sources of personalization 
information. Computers \& Education, 51(2), 776-786. https://doi.org/10.1016/j.compedu.2007.08.002

Van Zwanenberg, N., Wilkinson, L. J., \& Anderson, A. (2000). Felder and Silverman's Index of Learning Styles and Honey and Mumford's Learning Styles Questionnaire: how do they compare and do they predict academic performance? Educational Psychology, 20(3), 365-380. https://doi. org/10.1080/713663743

Vassileva, D., \& Bontchev, B. (2006). Self-adaptive hypermedia navigation based on learner model characters. http://www.iadat.org/iadat-e2006/ abstracts web/IADAT-e2006 13.pdf

Vermunt, J. D. (1998). The regulation of constructive learning processes. British journal of educational psychology, 68(2), 149-171. DOI: 10.1111/ j.2044-8279.1998.tb01281.x

Villaverde, J. E., Godoy, D., \& Amandi, A. (2006). Learning styles' recognition in e-learning environments with feed-forward neural networks. Journal of Computer Assisted Learning, 22(3), 197-206. DOI: 10.1111/j.13652729.2006.00169.x

Wang, T. I., Wang, K. T., \& Huang, Y. M. (2008). Using a style-based ant colony system for adaptive learning. Expert Systems with Applications, 34(4), 2449-2464. https://doi.org/10.1016/j. eswa.2007.04.014

Wen, D., Graf, S., Lan, C. H., Anderson, T., \& Dickson, K. (2007). Supporting web-based learning through adaptive assessment. FormaMente Journal, 2(1-2), 45-79. http://sgraf.athabascau. $\mathrm{ca} /$ publications/wen graf lan anderson kinshuk_dickson_FormaM̄Mentēournal.pdf

Xu, D., Wang, H., \& Su, K. (2002, January). Intelligent student profiling with fuzzy models. In System Sciences, 2002. HICSS. Proceedings of the 35th Annual Hawaii International Conference on (pp. 8-pp). IEEE. https://doi.org/10.1109/HICSS.2002.994005

Xu, D., \& Wang, H. (2006). Intelligent agent supported personalization for virtual learning environments. Decision Support Systems, 42(2), 825 843. https://doi.org/10.1016/j.dss.2005.05.033

Yang, T. C., Hwang, G. J., \& Yang, S. J. H. (2013). Development of an adaptive learning system with multiple perspectives based on students' learning styles and cognitive styles. Journal of Educational Technology \& Society, 16(4), 185. http://www.ifets.info/journals/16 4/15.pdf

Zakrzewska, D. (2010, June). Building group recommendations in e-learning systems. In KES International Symposium on Agent and Multi-Agent Systems: Technologies and Applications (pp. 391-400). Springer, Berlin, Heidelberg. https:// doi.org/10.1007/978-3-642-32066-8_7 